\title{
On a dividend problem with random funding
}

\author{
Josef Anton Strini ${ }^{1} \cdot$ Stefan Thonhauser ${ }^{1}$
}

Received: 21 January 2019 / Revised: 28 May 2019 / Accepted: 29 May 2019 / Published online: 13 June 2019

(c) The Author(s) 2019

\begin{abstract}
We consider a modification of the dividend maximization problem from ruin theory. Based on a classical risk process we maximize the difference of expected cumulated discounted dividends and total expected discounted additional funding (subject to some proportional transaction costs). For modelling dividends we use the common approach whereas for the funding opportunity we use the jump times of another independent Poisson process at which we choose an appropriate funding height. In case of exponentially distributed claims we are able to determine an explicit solution to the problem and derive an optimal strategy whose nature heavily depends on the size of the transaction costs. Furthermore, the optimal strategy identifies unfavourable surplus positions prior to ruin at which refunding is highly recommended.
\end{abstract}

Keywords Ruin theory $\cdot$ Classical risk model $\cdot$ Dividends $\cdot$ Stochastic control

\section{Introduction and some first considerations}

\subsection{Overview}

In this article we deal with an extension of the classical dividend maximization problem for an underlying classical (compound Poisson) surplus process. Our proposed extension considers a random funding opportunity which is modelled by the following procedure. The insurer actively searches for investors who are willing to provide additional funding for the insurance portfolio under consideration. If the search is successful, the insurer can choose the height of the funding, increase the surplus

Josef Anton Strini received support by the Austrian Science Fund (FWF) Single Project P26114.

Josef Anton Strini

j.strini@tugraz.at

Stefan Thonhauser

stefan.thonhauser@math.tugraz.at

1 Graz University of Technology, Institute of Statistics, Kopernikusgasse 24/III, $8010 \mathrm{Graz}$, Austria 
and possibly pay out higher dividends in the future. We model the search procedure by means of an intensity $\beta \geq 0$, such that the insurer finds funding opportunities at the jump-times of an additional and independent Poisson process. Naturally, new funding is costly and possibly external investors want to participate in future dividends. That is why we weight this additional capital with a factor $\phi \geq 1$ which plays the role of a proportional transaction cost. The corresponding value function of our problem is the difference of expected cumulated discounted dividends and weighted expected cumulated discounted funding, both up to the time of ruin. In Remark 2 we will discuss the point of view of an external investor and her participation in some detail.

For the case $\beta=0$ our approach just matches the classical dividend problem, i.e., no additional funding source can be found. Its treatment goes back to Gerber [6] and is analyzed in terms of optimal stochastic control by Azcue \& Muler [2, 3] and Schmidli [11]. The opposite extremal case $\beta \rightarrow \infty$ resembles the situation of a possible capital injection at any point in time, which is discussed in Sect. 4 . This problem is by now well known under the keywords maximal dividends and capital injections and was firstly formulated and solved by Kulenko \& Schmidli [9] with the subtle difference that the controlled surplus process is not allowed to get ruined and thus resulting in a different value function.

Certainly, the approach of interventions at the jump times of another process is related to the formulation of ruin theoretic problems under random observations. Such a model comprising dividends is introduced by Albrecher et al. [1] and gained some relevance in actuarial research over the last years. We need to emphasize that our present model is continuously monitored, i.e., dividend decisions can be made at any point in time and also the ruin event is immediately observed.

Another framework where dividend maximization problems for firm value determinations play a crucial role is corporate finance. There the underlying process, typically given by a diffusion process, is interpreted as a cash reservoir of a company and the expected value of cumulated dividends reflects the value of this company. The present question is studied in a similar fashion in this financial diffusion framework by Hugonnier et al. [7] in combination with an optimal stopping problem. As mentioned, the problem studied there is based on a continuous sample paths process and also the transaction cost parameter equals one, which results in a common single barrier type optimal strategy, both for dividends and fundings.

Interestingly, the recent paper by Zhang et al. [13] study a compound Poisson risk model with a particular capital injection procedure, which is very similar to the optimal one derived in our contribution. In contrast to our considerations, the focus is put on the determination of discounted penalty functions and dividend decisions are not part of the setup.

The paper is organized as follows. We start with the mathematical formulation of the model and associated stochastic optimization problem. In a next step we establish some basic properties of the value function and study parameter constellations which lead to degenerate optimal strategies. Having understood the crucial dependence on the magnitude of the transaction costs, we can subsequently determine the optimal strategy and corresponding value function. The key in this step is to prove the existence of a solution of a free-boundary value problem comprising two boundaries. We close the paper by 
some numerical illustrations which focus on the optimal strategy as a function of the transaction cost parameter $\phi$.

\subsection{Model setup}

In the subsequent lines we introduce the model of interest and the underlying stochastic protagonists. First of all we set up the stochastic basis of our considered model. We suppose a given probability space $(\Omega, \mathbf{F}, P)$ which carries the following underlying stochastic processes.

Let $N=\left(N_{t}\right)_{t \geq 0}$ be a Poisson process with intensity $\lambda>0$ and let $\left\{Y_{i}\right\}_{i \in \mathbb{N}}$ be a sequence of independent and identically distributed random variables with distribution function denoted by $F_{Y}$ with $F_{Y}(0)=0$, we set $\mathbb{E}\left(Y_{1}\right)=\mu$ and assume $N$ to be independent of $\left\{Y_{i}\right\}_{i \in \mathbb{N}}$. Then we consider the following compound Poisson process $S=\left(S_{t}\right)_{t \geq 0}$

$$
S_{t}=\sum_{i=1}^{N_{t}} Y_{i}
$$

which describes, as common in the classical risk model, the sum of all claims up to time $t$. Next we consider a jump process $B=\left(B_{t}\right)_{t \geq 0}$ with constant intensity $\beta$, i.e. a Poisson process, with which we are able to describe the occurrence times of new investors. In particular investors occur at the jump-times of $B$. Again, independence between $N, B,\left\{Y_{i}\right\}_{i \in \mathbb{N}}$ is assumed. Based on these ingredients we identify the filtration $\mathcal{F}=\left(\mathcal{F}_{t}\right)_{t \geq 0}$ which models the available information at time $t$. Consequently, we have to set

$$
\mathcal{F}_{t}=\sigma\left\{\mathcal{F}_{t}^{N}, \mathcal{F}_{t}^{B},\left\{Y_{1}, Y_{2}, \ldots, Y_{N_{t}}\right\}\right\} \cup \mathcal{N}
$$

where $\mathcal{F}^{N}$ and $\mathcal{F}^{B}$ are the filtrations generated by the respective processes and $\mathcal{N}$ denotes the sets of measure zero.

Assuming that the insurance company has an initial surplus $x_{0} \geq 0$ and receives premiums according to a rate $c$, we define the uncontrolled surplus or cash reserve process $X=\left(X_{t}\right)_{t \geq 0}$, by

$$
X_{t}=x_{0}+c t-S_{t} .
$$

The control processes for the state process $X$ are on the one hand the dividend process $L=\left(L_{t}\right)_{t \geq 0}$, an adapted and càglàd process, hence it is previsible, which is increasing and fulfills $L_{0} \equiv 0$. It represents the cumulated dividends up to time t. On the other hand we consider the control process $f=\left(f_{t}\right)_{t \geq 0}$, previsible as well, and $P$ - almost surely non-negative, i.e. $f_{t} \geq 0 P-$ a.s. The control $f$ corresponds to the magnitude of the new funding at time $t$ in case $B$ jumps. According to that, the controlled cash reserve process reads as follows

$$
X_{t}^{L, f}=x_{0}+c t-S_{t}-L_{t}+\int_{0}^{t} f_{s} d B_{s} .
$$


In our setting it is not allowed that ruin is induced by dividend payments and therefore the relation

$$
X_{t+}^{L, f}=X_{t}^{L, f}-\Delta L_{t} \geq 0
$$

has to hold $P-$ a.s.

Remark 1 Due to the independence assumptions we have that $P-$ a.s. the two Poisson processes $N$ and $B$ do not jump at the same time. Since the paths of the dividend process $L$ are left-continuous one needs to read $\Delta L_{t}=L_{t+}-L_{t}$.

\subsection{Optimization problem and value function}

The stated aim in our setting is to find the optimal combined dividend and funding strategy which maximizes the expected cumulated discounted future dividends deducting at least the received total additional funding. The deduction depends on a proportional funding or transaction cost parameter denoted by $\phi \geq 1$. Concerning transaction costs in the classical capital injection problem one could think of implementation costs as common in mathematical finance. On the other hand if we think of external funding as considered in our model we could interpret transaction costs as some kind of commission or costs for intermediation. Hence the value function is defined by

$$
V(x)=\sup _{(L, f) \in \Theta} \mathbb{E}_{x}\left[\int_{0}^{\tau^{L, f}} e^{-\delta t} d L_{t}-\phi \int_{0}^{\tau^{L, f}} e^{-\delta t} f_{t} d B_{t}\right],
$$

here $\tau^{L, f}$ denotes the first time when the controlled cash reserve process becomes negative, namely $\tau^{L, f}=\inf \left\{t \geq 0 \mid X_{t}^{L, f}<0\right\}$ and $\Theta$ is the set containing those admissible processes $(L, f)$ such that

$$
\mathbb{E}_{x}\left[\int_{0}^{\tau^{L, f}} e^{-\delta t}\left(d L_{t}+\phi f_{t} d B_{t}\right)\right]<\infty
$$

Remark 2 (Injection versus Funding) In the initial contribution by Kulenko \& Schmidli [9] new capital for the insurance company is provided by shareholders with the ultimate goal to prevent ruin. In this context the arising problem is considered to be the classical injection problem. In our contribution we have chosen the term funding for additional capital on purpose to point out that ruin is still possible in our model set up. This is in contrast to the classical motivation of capital injections. The way of incorporating additional capital was also mentioned in Hugonnier et al. [7] in the context of corporate finance, especially it is discussed in detail in the Supplementary Appendix [8]. In this case the additional capital is supposed to be provided by external investors and not initial shareholders as suggested in injection problems. Consequently, one needs to discuss the share of old and new investors on the firm value's gain due to new investments. Such questions of allocating a commonly generated surplus are heavily studied under the name bargaining problems 
which are part of game theory. An overview of such problems and optimal allocation rules is Binmore et al. [5] for instance. In our problem, as also used in [8], we have that the gain of an injection $f$, measured by future expected dividends, is

$$
\mathcal{G}_{f} V(x):=V(x+f)-\phi f-V(x) .
$$

Now, as stated in [5] an agreeable split of the gain is the subject of negotiations between the two investors. The results from [5] show that an equilibrium split of gain is achieved by identifying the new investors part $C^{*}$ from

$$
C^{\eta}\left(\mathcal{G}_{f} V(x)-C\right)^{1-\eta} \mapsto \max _{C \geq 0}
$$

The power $\eta \in(0,1)$ is typically interpreted as bargaining power, see [5, Section 4]. Its size can be linked to the magnitude of the arrival intensity of new investors $\beta$. For example $\eta$ can be a decreasing function of $\beta$, i.e., very frequent arrivals of new investors decrease their bargaining power. One can derive that the maximal share in our model is given by $C_{f}^{*}=\eta \mathcal{G}_{f} V(x)$. Therefore, if we assume the additional capital to be externally provided, we need to alter the value function of the initial investors. From their perspective the dividends need to be reduced by funding $f$ and the new investors share $C_{f}^{*}$. This leads to an implicitly defined value function $V^{I}$ which is given by

$$
V^{I}(x)=\sup _{(L, f) \in \Theta} \mathbb{E}_{x}\left[\int_{0}^{\tau^{L, f}} e^{-\delta t} d L_{t}-\int_{0}^{\tau^{L, f}} e^{-\delta t}\left(\phi f_{t}+\eta \mathcal{G}_{f_{t}} V^{I}\left(X_{t-}^{L, f}\right)\right) d B_{t}\right] .
$$

At this point we can follow the arguments as presented in [8, Section H]. Namely, it can be shown by martingale arguments that if we have identified $V$ from (1) and optimal controls $L^{*}, f^{*}$ for a modified arrival intensity of $\beta(1-\eta)$ of $B$ and otherwise unchanged parameters (referring to a new measure $\tilde{P}$ ) one has a solution to (2) under jump intensity $\beta$ of $B$ (under the original measure $P$ ).

If we assume that the controls are constant and the dividend control suffices $d L_{t}=l d t$ for some $l \in \mathbb{R}^{+}$, we face a Markov process whose infinitesimal generator is

$$
\mathcal{A}^{l, f} g(x)=c g^{\prime}(x)-l g^{\prime}(x)+\beta[g(x+f)-g(x)]+\lambda \int_{0}^{\infty}[g(x-y)-g(x)] d F_{Y}(y) .
$$

Naturally, the function $g$ above has to be in the domain of the generator $\mathcal{D}\left(\mathcal{A}^{l, f}\right)$, which contains absolutely continuous functions $h$ satisfying an integrability condition $\mathbb{E}\left[\left|h\left(X_{t}^{l . f}\right)\right|\right]<\infty$, see Rolski et al. [10, Th. 11.2.2]. Using this expression we can state the Hamilton-Jacobi-Bellman equation of this problem

$$
\begin{aligned}
\max & \left\{c g^{\prime}(x)-(\lambda+\delta) g(x)+\lambda \int_{0}^{x} g(x-y) d F_{Y}(y)\right. \\
& \left.+\beta \sup _{f \geq 0}\{g(x+f)-g(x)-\phi f\}, 1-g^{\prime}(x)\right\}=0 .
\end{aligned}
$$


From the shape of the HJB-equation we can immediately derive some properties of its solutions.

Lemma 1 Let $g$ be a continuously differentiable solution to the HJB-equation (3), then $g$ is strictly monotone increasing $\left(g^{\prime} \geq 1\right)$ and bounded from below by $\frac{c}{\lambda+\delta}>0$.

Proof From the equation we directly obtain that $g^{\prime} \geq 1$ and if we consider the limit $x \searrow 0$ we get $g(0+) \geq \frac{c}{\delta+\lambda}>0$. Since $g$ is monotone increasing and continuous the assertion follows.

Furthermore, we can bound the value function from below similarly as done by Azcue \& Muller [2] or by Schmidli [11, p. 80 Lemma 2.37].

Lemma 2 In the present model setup the value function fulfills

$$
V(x) \geq x+\frac{c}{\lambda+\delta} .
$$

Proof For the special choice $(L, f) \equiv(L, 0)$ we face an admissible dividend strategy for the original dividend maximization problem. The bound follows from the above cited (by now classical) results.

\section{Solution of the optimization problem}

In the following we assume that the claim size distribution coincides with an exponential distribution with parameter $\alpha$. We try to identify an optimal strategy and determine an explicit solution to the problem. In case of an arbitrary claim size distribution one can expect a strategy of band type to be optimal. One needs to mention that the presence of the financing control complicates the situation in comparison to other modifications of the dividend problem with exponentially distributed claims in the literature.

We start with identifying parameter sets which lead to somehow degenerate optimal strategies.

\subsection{Optimality of keeping the reserve at zero}

For a special parameter configuration we obtain that the optimal strategy is to payout the initial reserve immediately and keep on paying dividends such that the current reserve remains zero, which means that the dividend rate is $c$ and the first claim causes ruin. Compare to classical results as presented in [11, p. 93].

Lemma 3 The optimal strategy is to payout immediately the initial reserve and then payout dividends at the premium rate $c$ if $(\delta+\lambda)^{2} \geq c \alpha \lambda$. Consequently, the value function has the following form 


$$
V(x)=x+\frac{c}{\lambda+\delta} .
$$

Proof Using $V(x)=x+\frac{c}{\lambda+\delta}$ the proof is analogous to the one given in [11, p. 93]. Just note that in the present problem with capital supply the additional part of the HJB-equation corresponding to $f$ is zero,

$$
\beta \sup _{f \geq 0}\{V(x+f)-V(x)-\phi f\}=\beta \sup _{f \geq 0}\{(1-\phi) f\}=0,
$$

since $\phi \geq 1$.

From the latter result we see that we need to focus on $(\delta+\lambda)^{2}<c \alpha \lambda$, which in turn implies that $c \alpha>\delta+\lambda$, since we assume that all parameters are positive.

\subsection{An embedded problem}

At the outset of tackling the problem we try as first conjectures some common types of controls such as barrier and simple band strategies. It turned out that they can not be optimal in general. Therefore, in order to get an idea of the shape of the optimal strategy we exploit a numerical approach.

At first fix $n \in \mathbb{N}$ and allow for at most $n$ capital injections (at the jump times $Z_{1}, \ldots, Z_{n}$ of $\left.B\right)$, the corresponding family of value functions is defined by

$$
V_{n}(x)=\sup _{(L, f) \in \Theta} \mathbb{E}\left[\int_{0}^{\tau^{L, f}} e^{-\delta s} d L_{s}-\phi \sum_{i=1}^{B_{\tau} L, f \wedge n} e^{-\delta Z_{i}} f_{Z_{i}}\right] .
$$

One may notice that $V_{0}$ is the value function of the classical dividend maximization problem and in the situation of exponentially distributed claims is explicitly known. However, the method below does not need to assume exponential claims but is focused on barrier type dividend strategies, such a strategy at level $b$ says that every excess of $b$ is immediately distributed as dividends. Certainly, this can be generalized.

We have that $V_{n}$ after using one intervention restarts with $V_{n-1}$, which can be used when maximizing with respect to $f$. The deduced numerical procedure is as follows:

1. Compute $V_{0}$ without additional capital $(f=0)$, by solving

$$
0=c g^{\prime}(x)-(\delta+\lambda) g(x)+\lambda \int_{0}^{x} g(x-y) d F_{Y}(y),
$$

for $0 \leq x \leq b$ and $g^{\prime}(x)=1$ for $x>b$. If the optimal $b$ is not known one can do this for different values of $b$. Choosing the maximizing $b$, we obtain an approximation to $V_{0}$ with optimal barrier, say $b_{0}^{*}$. Then we can compute the optimal state dependent $f_{0}(x)$ by setting 


$$
f_{0}=\underset{f \geq 0}{\operatorname{argmax}}\left\{V_{0}(x+f)-V_{0}(x)-\phi f\right\} .
$$

2. Compute $V_{1}$, where we allow for one financial injection, exactly $f_{0}$, at the first jump time of $\left(B_{t}\right)_{t \geq 0}$ and solve for different values of $b$

$$
0=c g^{\prime}(x)-(\delta+\lambda) g(x)+\lambda \int_{0}^{x} g(x-y) d F_{Y}(y)+\beta\left[V_{0}\left(x+f_{0}\right)-V_{0}(x)-\phi f_{0}\right],
$$

for $0 \leq x \leq b$ and $g^{\prime}(x)=1$ for $x>b$. The usage of the maximizing $b$ results in an approximation of $V_{1}$. As next step replace $V_{0}$ by $V_{1}$ in the first step and go on with computing $V_{2}$, where $f_{1}$ is obtained according to step 1 . Consequently, at the first jump time of $B_{t}$ we use $f_{1}$, then we switch to the problem with one funding for which the value is given by $V_{1}$.

Of course we have to choose in every step the best value for the threshold $b$, which is additionally illustrated in the plot below. There $V_{(0)}(x)$ denotes the numerical solution of the usual dividend problem, further $V_{(i)}(x ; b)$ denotes the solution of the iteration, when $i \in\{1,2, \ldots\}$ funding opportunities are allowed and the barrier is chosen to be $b$ (Fig. 1).

This approach reveals a new type of possibly optimal strategy to us, which turns out to be the right conjecture.

\subsection{Resulting new strategy}

Using the results from the numerical approach, we are able to construct a new strategy for our problem. The new strategy is of band type and specified by two parameters $0 \leq a \leq b<\infty$ such that

- the dividend strategy is of barrier type at level $b$,

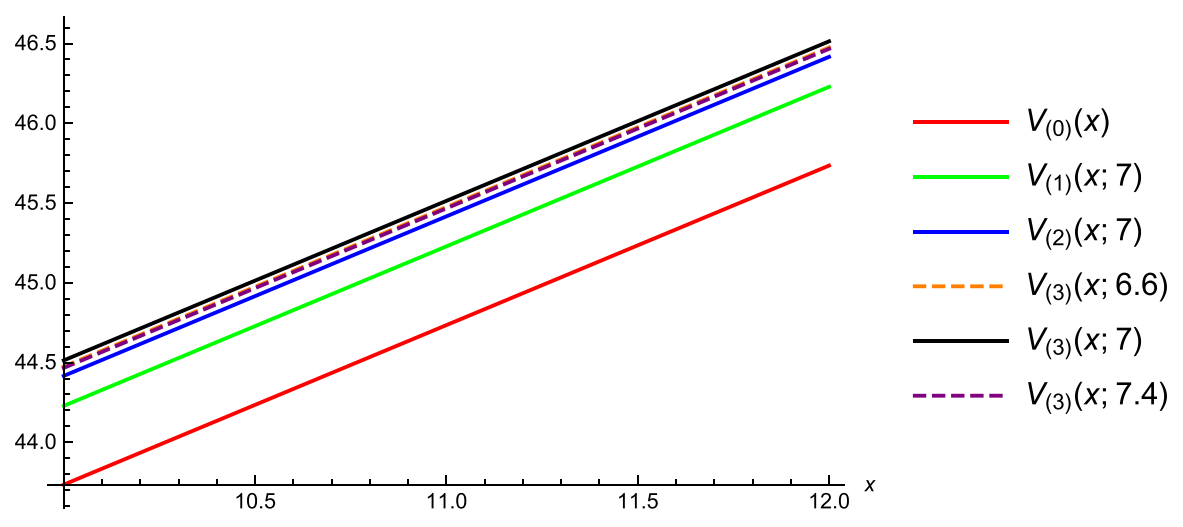

Fig. 1 Comparison of different iterative solutions 


$$
\begin{aligned}
\Delta L_{0+} & =(x-b) I_{\{x>b\}} \\
d L_{t} & =c I_{\left\{X_{t-}^{L, f}=b\right\}} d t, \quad t>0,
\end{aligned}
$$

- the financing strategy only applies at reserve levels $x \in[0, a)$. It is given by $f(x)=(a-x) I_{\{0 \leq x<a\}}$, with the feature that only below level $a$ we search for a funding source. If one appears, we choose the funding height to such an extent that the surplus jumps up to $a$ and in general not to the barrier level $b$.

For initial surplus $x \geq 0$ we denote the value, i.e., performance function, according to such a strategy by $V(x ; a, b)$. By construction it makes sense to write this function in the following form:

$$
V(x ; a, b)=\left\{\begin{array}{lll}
V_{l}(x ; a, b), & \text { if } & 0 \leq x \leq a, \\
V_{u}(x ; a, b), & \text { if } & a \leq x \leq b, \\
x-b+V(b ; a, b), & \text { if } & x>b .
\end{array}\right.
$$

Whereby, using Dynkin-formula type arguments or classical arguments based on conditioning on the first claim occurence, the functions $V_{l}(x)=V_{l}(x ; a, b)$ and $V_{u}(x)=V_{u}(x ; a, b)$ have to fulfill the equations

$$
\begin{gathered}
c V_{l}^{\prime}(x)-(\delta+\lambda) V_{l}(x)+\lambda \int_{0}^{x} V_{l}(x-y) \alpha e^{-\alpha y} d y \\
+\beta\left(V_{l}(a)-V_{l}(x)-\phi(a-x)\right)=0, \\
c V_{u}^{\prime}(x)-(\delta+\lambda) V_{u}(x)+\lambda \int_{0}^{x-a} V_{u}(x-y) \alpha e^{-\alpha y} d y \\
+\lambda \int_{x-a}^{x} V_{l}(x-y) \alpha e^{-\alpha y} d y=0, \\
V_{l}(a)=V_{u}(a) \text { and } V_{u}^{\prime}(b)=1 .
\end{gathered}
$$

We get immediately, using the above equations, that $V(x ; a, b)$ is continuously differentiable in $x$. Furthermore, we obtain that continuity implies differentiability, i.e. $V_{l}^{\prime}(a)=V_{u}^{\prime}(a)$ if and only if $V_{l}(a)=V_{u}(a)$. This means that the condition $V_{l}(a)=V_{u}(a)$ in $(7)$ is equivalent to the condition $V_{l}^{\prime}(a)=V_{u}^{\prime}(a)$.

We use the method of equating coefficients in order to solve the above equations (5), (6) and (7) explicitely. This yields functions

$$
\begin{gathered}
V_{l}(x ; a, b):=A_{1}(a, b) e^{R_{1} x}+A_{2}(a, b) e^{R_{2} x}+A_{3}(a, b) x+A_{4}(a, b), \\
V_{u}(x ; a, b):=B_{1}(a, b) e^{S_{1} x}+B_{2}(a, b) e^{S_{2} x},
\end{gathered}
$$


where $S_{1}<0<S_{2}$ are solutions to $c S-(\delta+\lambda)+\frac{\alpha \lambda}{\alpha+S}=0$. The exponents $R_{1}<0<R_{2}$ solve $c R-(\delta+\lambda+\beta)+\frac{\alpha \lambda}{\alpha+R}=0$. Note that under our assumptions we have that $S_{1}+S_{2}<0$. The coefficients $A_{1}, \ldots, B_{2}$ are obtained by a system of six linear equations and do heavily depend on the parameters $a, b$.

We observe that numerical maximization of the function $V(x ; a, b)$ in $a$ and $b$ for fixed $x$ results in levels $a^{*}$ and $b^{*}$ which are independent of $x$. These particular levels also coincide with the solutions of second order smooth fit conditions in $a$ and $b$. Additionally, we need to fulfill these second order smooth fit conditions in order to get a function which is twice continuously differentiable. This seems to be superfluous, since the domain of the generator only asks for absolute continuity, but in combination with concavity it serves as a basis for a direct proof of the associated verification theorem. The conditions read as follows:

$$
F(a, b)=\left(\begin{array}{c}
\left.\frac{\partial^{2}}{\partial x^{2}} V_{u}(x ; a, b)\right|_{x=b} \\
{\left.\left[\frac{\partial^{2}}{\partial x^{2}} V_{l}(x ; a, b)-\frac{\partial^{2}}{\partial x^{2}} V_{u}(x ; a, b)\right]\right|_{x=a}}
\end{array}\right) \stackrel{!}{=}\left(\begin{array}{l}
0 \\
0
\end{array}\right) .
$$

In Fig. 2 the green surface corresponds to $\left.\frac{\partial^{2}}{\partial x^{2}} V_{u}(x ; a, b)\right|_{x=b}$, the orange surface corresponds to $\left.\frac{\partial^{2}}{\partial x^{2}} V_{l}(x ; a, b)\right|_{x=a}-\left.\frac{\partial^{2}}{\partial x^{2}} V_{u}(x ; a, b)\right|_{x=a}$ and the blue plane highlights the zero level. The red curves mark the intersection of the two surfaces with the zero level.

Finally, we have to prove the existence of thresholds $a^{*}$ and $b^{*}$ such that the smooth fit conditions are fulfilled. In our treatment we are able to derive an interesting condition, which turns out to be equivalent to one of the conditions above.

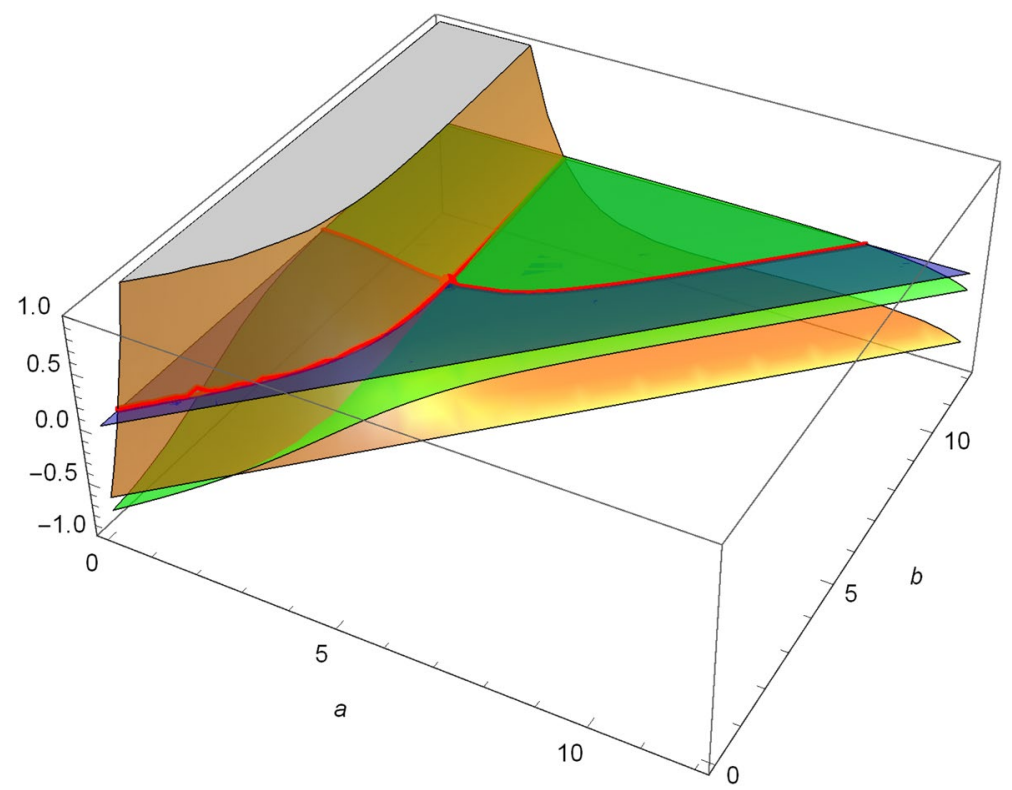

Fig. 2 Illustration of the problem of finding a solution $\left(a^{*}, b^{*}\right)$ to (10) 
If we differentiate the two integro-differential equations (for $x \in(0, a)$ and $x \in(a, b))$, which characterize $V_{l}$ and $V_{u}$, we obtain

$$
c V_{l}^{\prime \prime}(x)-(\delta+\lambda+\beta) V_{l}^{\prime}(x)+\lambda \alpha e^{-\alpha x} V_{l}(0)+\lambda \int_{0}^{x} V_{l}^{\prime}(x-y) \alpha e^{-\alpha y} d y+\beta \phi=0
$$

and

$$
\begin{aligned}
& c V_{u}^{\prime \prime}(x)-(\delta+\lambda) V_{u}^{\prime}(x)+\underbrace{\lambda \alpha e^{-\alpha(x-a)} V_{u}(a)-\lambda \alpha e^{-\alpha(x-a)} V_{l}(a)}_{=0} \\
& \quad+\lambda \int_{0}^{x-a} V_{u}^{\prime}(x-y) \alpha e^{-\alpha y} d y+\lambda \alpha e^{-\alpha x} V_{l}(0)+\lambda \int_{x-a}^{x} V_{l}^{\prime}(x-y) \alpha e^{-\alpha y} d y=0 .
\end{aligned}
$$

If we now set $x=a$ and calculate the difference of those two equations, we get using $V_{l}^{\prime}(a)=V_{u}^{\prime}(a)$ and $V_{l}(a)=V_{u}(a)$ that

$$
c\left(V_{l}^{\prime \prime}(a)-V_{u}^{\prime \prime}(a)\right)=\beta\left(V_{l}^{\prime}(a)-\phi\right) .
$$

Hence we have that $V_{l}^{\prime \prime}(a)=V_{u}^{\prime \prime}(a)$ if and only if $V_{l}^{\prime}(a)=\phi$.

Remark 3 If we consider the following part of the HJB-equation:

$$
\sup _{f \geq 0}\left\{V_{l}(x+f)-V_{l}(x)-\phi f\right\},
$$

we obtain for the function $V_{l}(x)$, if it is concave, that the term inside the supremum is maximal if $V_{l}^{\prime}(x+f)=\phi$. This means that using an $a$ such that $a=f+x=V_{l}^{\prime[-1]}(\phi)$, yields that the corresponding term $\left(V_{l}^{\prime}(a)-\phi\right)$ in the equation above is zero.

\subsubsection{Extremal behaviour of the optimal strategy}

First of all we want to know, whether the optimal strategy uses the additional funding or not, which means that we have to determine the reasons for the choice $a^{*}=0$. For that purpose we consider the solution of the usual dividend problem, which is well-known in the literature, see [11],

$$
\tilde{V}(x ; b)= \begin{cases}\frac{h(x)}{h^{\prime}(b)}, & \text { if } \quad 0 \leq x \leq b \\ x-b+\tilde{V}(b ; b), & \text { if } \quad x>b .\end{cases}
$$

Here

$$
h(x)=e^{S_{1} x}\left(S_{1}+\alpha\right)-e^{S_{2} x}\left(S_{2}+\alpha\right)
$$

where $S_{1}$ and $S_{2}$ are the same exponents as before and the optimal barrier ensuring twice continuous differentiability of the value function has the following form 


$$
\tilde{b}=\ln \left(\frac{S_{2}^{2}\left(S_{2}+\alpha\right)}{S_{1}^{2}\left(S_{1}+\alpha\right)}\right) \frac{1}{S_{1}-S_{2}} .
$$

In this case we have to assume that

$$
(\delta+\lambda)^{2}<c \alpha \lambda,
$$

in order to make sure that $\tilde{b}>0$ holds. This yields that in the usual dividend problem $\tilde{V}(x ; \tilde{b})$ is the value function. We have that $\tilde{V}(x ; b)=V(x ; 0, b)$.

Remark 4 At first one notices that

$$
\tilde{V}^{\prime}(0 ; \tilde{b})=\frac{\left(S_{1}-S_{2}\right)\left(\alpha+S_{1}+S_{2}\right)}{S_{1}\left(\alpha+S_{1}\right)\left(\frac{S_{2}^{2}\left(\alpha+S_{2}\right)}{S_{1}^{2}\left(\alpha+S_{1}\right)}\right)^{\frac{S_{1}}{S_{1}-S_{2}}}-S_{2}\left(\alpha+S_{2}\right)\left(\frac{S_{2}^{2}\left(\alpha+S_{2}\right)}{S_{1}^{2}\left(\alpha+S_{1}\right)}\right)^{\frac{S_{2}}{S_{1}-S_{2}}}} \geq 1
$$

A second thought concerns the behaviour of a possibly optimal level $a$ with respect to the parameter $\phi$. The following is more a heuristic intuition than a rigorous treatment. But if we consider $a=x+f^{*}=g^{\prime[-1]}(\phi)$, where $f^{*}$ is the maximizing argument of the supremum part of the HJB-equation

$$
\sup _{f \geq 0}\{g(x+f)-g(x)-\phi f\}
$$

as mentioned in the previous remark, we get (considering $a$ as a function of $\phi$, i.e. $\left.a(\phi)=g^{[-1]}(\phi)\right)$ that

$$
a^{\prime}(\phi)=\left(g^{[-1]}\right)^{\prime}(\phi)=\frac{1}{g^{\prime \prime}\left(g^{\prime[-1]}(\phi)\right)}<0,
$$

if we assume that $g$ is concave, which will be true if $g$ is the value function. This means that the lower threshold $a$ is decreasing in $\phi$.

Proposition 1 Let $(\delta+\lambda)^{2}<c \alpha \lambda$, such that the optimal barrier in the classical dividend problem $\tilde{b}$ is positive. For the optimal levels $0 \leq a^{*} \leq b^{*}$ in the dividend problem with random funding we obtain that $a^{*}=0$ and $b^{*}=\tilde{b}$ if and only if $\phi \geq \tilde{V}^{\prime}(0 ; \tilde{b})$.

This means that for $\phi \geq \tilde{V}^{\prime}(0 ; \tilde{b})$ and $(\delta+\lambda)^{2}<c \alpha \lambda$ the simple barrier strategy is optimal, and the solution of the classical dividend problem coincides with the solution of the extended problem.

Proof We assume that $\phi \geq \tilde{V}^{\prime}(0 ; \tilde{b})$ and have to show that $a^{*}=0$ and $b^{*}=\tilde{b}$ are the optimal thresholds so that $V(x ; 0, \tilde{b})=\tilde{V}(x ; \tilde{b})$ solves the HJB-equation, is concave and $\mathcal{C}^{2}$ - the ingredients we later need in the verification theorem. 
We know that this candidate function is concave, $\mathcal{C}^{2}$ and solves the HJB-equation of the classical dividend problem with $\beta=0$. Using this, we obtain for $0 \leq x<\tilde{b}$ that

$$
1-\tilde{V}^{\prime}(x ; \tilde{b})<0 .
$$

For the first part of the HJB-equation it remains to show that

$$
\beta \sup _{f \geq 0}\{\tilde{V}(x+f ; \tilde{b})-\tilde{V}(x ; \tilde{b})-\phi f\}=0 .
$$

If we choose $f=0$ the term inside the supremum is zero. Otherwise, if $f>0$ we obtain that

$$
\tilde{V}(x+f ; \tilde{b})-\tilde{V}(x ; \tilde{b})-\phi f<0 .
$$

This holds true because if we use $\tilde{V}^{\prime \prime}(x ; \tilde{b})<0$ we get

$$
\frac{\tilde{V}(x+f ; \tilde{b})-\tilde{V}(x ; \tilde{b})}{(x+f)-x} \leq \tilde{V}^{\prime}(x ; \tilde{b})<\tilde{V}^{\prime}(0 ; \tilde{b})
$$

and from $\phi \geq \tilde{V}^{\prime}(0 ; \tilde{b})$, the above inequality follows. For the values $x \geq \tilde{b}$ we know that $\tilde{V}^{\prime}(x ; \tilde{b})=1$, and that the first part of the HJB-equation (which coincides with the first part of the classical HJB-equation) is negative. So it remains to check whether

$$
\beta \sup _{f \geq 0}\{\tilde{V}(x+f ; \tilde{b})-\tilde{V}(x ; \tilde{b})-\phi f\} \leq 0
$$

holds. But this is true since if we plug in the linear function for $x>\tilde{b}$ we get that $\beta \sup _{f \geq 0}\{(1-\phi) f\} \leq 0$, since $\phi \geq 1$. The special case $x=\tilde{b}$ is analogue to the situation $x<\tilde{b}$.

For the other direction, if $a^{*}=0$ and $b^{*}=\tilde{b}$ are optimal, we have to show $\phi \geq \tilde{V}^{\prime}(0 ; \tilde{b})$. For that purpose we assume the opposite, namely that $\phi<\tilde{V}^{\prime}(0 ; \tilde{b})$. But in this case we can exploit the fact that $\tilde{V}^{\prime}(\tilde{b} ; \tilde{b})=1$ and $\tilde{V}^{\prime \prime}(x ; \tilde{b})<0 \forall x \in(0, \tilde{b})$, in addition to the above assumption $\tilde{V}^{\prime}(0 ; \tilde{b})>\phi \geq 1$. Putting this together yields by the intermediate value theorem that $\exists ! \bar{x} \in(0, \tilde{b}): \tilde{V}^{\prime}(\bar{x} ; \tilde{b})=\phi$. We want to show that

$$
\beta \sup _{f \geq 0}\{\tilde{V}(x+f ; \tilde{b})-\tilde{V}(x ; \tilde{b})-\phi f\}>0 .
$$

Differentiating the inner term and setting it equal to zero yields that

$$
\tilde{V}^{\prime}(x+f ; \tilde{b}) \stackrel{!}{=} \phi,
$$

so we obtain that $f^{*}=\bar{x}-x$ which is positive, if $x \in(0, \bar{x})$. Applying Taylor's formula gives for some $\theta \in(x, \bar{x})$ the following 


$$
\begin{aligned}
\tilde{V}(x ; \tilde{b}) & =\tilde{V}(\bar{x} ; \tilde{b})+\tilde{V}^{\prime}(\bar{x} ; \tilde{b})(x-\bar{x})+\frac{1}{2} \tilde{V}^{\prime \prime}(\theta ; \tilde{b})(x-\bar{x})^{2} \\
& =\tilde{V}(\bar{x} ; \tilde{b})-\phi(\bar{x}-x)+\frac{1}{2} \tilde{V}^{\prime \prime}(\theta ; \tilde{b})(x-\bar{x})^{2} \\
& <\tilde{V}(\bar{x} ; \tilde{b})-\phi(\bar{x}-x) .
\end{aligned}
$$

Finally we obtain that this function does not solve the HJB-equation and $a^{*}=0$ being optimal cannot work out, which is a contradiction.

The above proposition gives us the optimal strategy in the case $\phi \geq \tilde{V}^{\prime}(0, \tilde{b})$. Furthermore, only in that case the usual dividend barrier strategy is optimal. In the next step we consider the lowest bound for the parameter $\phi$ where a non-trivial strategy appears, namely $\phi=1$.

Lemma 4 Let $(\delta+\lambda)^{2}<c \alpha \lambda$. For the optimal levels $a^{*} \leq b^{*}$ for the dividend problem with random funding we obtain that $a^{*}=b^{*}$ if $\phi=1$.

In this case we are in the following situation: if an investor occurs we generate external funding to such an extent that we arrive with the surplus process at the dividend barrier, which triggers dividend payments. Hence, there is no gap between the dividend barrier and the funding level. In contrast to the setting by Kulenko \& Schmidli [9] where for $\phi=1$ it is optimal on the one hand to consume the initial capital and all premiums as dividends and on the other hand injections can be chosen to compensate all claims, we get the extremal solution $a^{*}=b^{*}>0$. This is due to the presence of the ruin event in our setting, whereas in [9] injections need to be used to "repair" possible ruin events. The feature $a^{*}=b^{*}$ for $\phi=1$ shows that "cheap" funding is exploited in full extent to speed up dividend payments.

Proof Solving the above equations for $a=b$, we get the function $V_{l}(x ; a, a)$. It remains to prove the existence of $a^{*}=b^{*}$, resulting in $V_{l}\left(x ; a^{*}, b^{*}\right)$, such that the assumptions of the verification theorem are fulfilled. At this point we know that $V_{l}^{\prime}(a ; a, a)=1$ and we have to find $a$ such that the smooth fit condition is fulfilled: $M(a):=V_{l}^{\prime \prime}(a ; a, a) \stackrel{!}{=} 0$. Evaluating this function at $a=0$ yields $M(0)=\frac{(\delta+\lambda)^{2}-c \alpha \lambda}{c(\delta+\lambda)}$, which is negative according to our assumptions. Otherwise, we would have a value function of the form $V(x)=x+\frac{c}{\delta+\lambda}$ as treated in the corresponding lemma above. Furthermore, if $M(0)=0$ we obtain that $a^{*}=b^{*}=0$. This is also in line with the just mentioned case of a linear value function.

On the other hand we know that

$$
M(a)=V_{l}^{\prime \prime}(a ; a, a)=A_{1}(a, a) e^{R_{1} a}+A_{2}(a, a) e^{R_{2} a},
$$


is continuous and $\lim _{a \rightarrow \infty} M(a)=R_{2} \frac{\delta}{\beta+\delta}$ is strictly positive. This yields that there exists an $a^{*}$ such that $M\left(a^{*}\right)=0$. If there would be more than one point, such that the smooth fit conditions are fulfilled, we decide to choose the smallest one. At this $a^{*}>0$ we are able to exploit the equations $V_{l}^{\prime}\left(a^{*} ; a^{*}, a^{*}\right)=1$ and $V_{l}^{\prime \prime}\left(a^{*} ; a^{*}, a^{*}\right)=0$ to get that $A_{1}\left(a^{*}, a^{*}\right)<0$ and $A_{2}\left(a^{*}, a^{*}\right)>0$. This yields that $V_{l}^{\prime \prime \prime}\left(x ; a^{*}, a^{*}\right)>0$ for all $x \geq 0$, moreover together with $V_{l}^{\prime \prime}\left(a^{*} ; a^{*}, a^{*}\right)=0$ we obtain that $V_{l}^{\prime \prime}\left(x ; a^{*}, a^{*}\right)<0$ for $0 \leq x<a^{*}$. Which in turn implies that $V_{l}^{\prime}\left(x ; a^{*}, a^{*}\right)>1$ for $0 \leq x<a^{*}$. So the obtained function

$$
V(x)=V\left(x ; a^{*}, a^{*}\right)= \begin{cases}V_{l}\left(x ; a^{*}, a^{*}\right), & \text { if } \quad 0 \leq x \leq a^{*}, \\ x-a^{*}+V\left(a^{*} ; a^{*}, a^{*}\right), & \text { if } \quad x>a^{*} .\end{cases}
$$

is twice continuously differentiable and concave, in addition to that it fulfills the HJB-equation with $\phi=1$. Altogether, this enables us to apply the verification theorem.

\subsubsection{The case of moderate $\phi$}

Up to now we have investigated the following cases and obtained in each case the optimal combined strategy:

- $\phi \geq \tilde{V}^{\prime}(0 ; \tilde{b}) \Rightarrow a^{*}=0$ and $b^{*}=\tilde{b}$,

- $\phi=1 \Rightarrow a^{*}=b^{*}$.

In this section we fill the missing gaps in order to have an optimal solution for every admissible value of $\phi \geq 1$.

Theorem 1 For $1<\phi<\tilde{V}^{\prime}(0 ; \tilde{b})$ and $(\delta+\lambda)^{2}<c \alpha \lambda$, we have that there exist $0<a^{*}<b^{*}$ such that the smooth fit conditions (12) and (13) are satisfied. The resulting function $V\left(x ; a^{*}, b^{*}\right)$ from (4) is a twice differentiable and concave solution to the HJB-equation (3).

Proof Obviously, we have to solve the equations related to our band strategy for $1<\phi<\tilde{V}^{\prime}(0, \tilde{b})$. This leads to a solution heavily depending on $a \leq b$ as in the previous case and it remains to choose the values $a$ and $b$ such that the equivalent smooth fit conditions are fulfilled, which are restated here

$$
\begin{aligned}
& V_{u}^{\prime}(a)=B_{1}(a, b) S_{1} e^{S_{1} a}+B_{2}(a, b) S_{2} e^{S_{2} a} \stackrel{!}{=} \phi, \\
& V_{u}^{\prime \prime}(b)=B_{1}(a, b) S_{1}^{2} e^{S_{1} b}+B_{2}(a, b) S_{2}^{2} e^{S_{2} b \stackrel{!}{=}} 0 .
\end{aligned}
$$

Anyway, the coefficients $B_{1}(a, b)$ and $B_{2}(a, b)$ are fixed such that 


$$
V_{u}^{\prime}(b)=B_{1}(a, b) S_{1} e^{S_{1} b}+B_{2}(a, b) S_{2} e^{S_{2} b}=1,
$$

holds true. Transforming this equation twice, leads to

$$
\begin{aligned}
& B_{1}(a, b) S_{1}^{2} e^{S_{1} b}=S_{1}\left(1-B_{2}(a, b) S_{2} e^{S_{2} b}\right), \\
& B_{1}(a, b) S_{1} e^{S_{1} a}=\left(1-B_{2}(a, b) S_{2} e^{S_{2} b}\right) e^{S_{1}(a-b)} .
\end{aligned}
$$

Now we insert this expression into the equations (12) and (13) and obtain

$$
\begin{aligned}
& B_{2}(a, b) S_{2}\left(e^{S_{2} a}-e^{S_{2} b+S_{1}(a-b)}\right) \stackrel{!}{=} \phi-e^{S_{1}(a-b)}, \\
& B_{2}(a, b) S_{2}\left(e^{S_{2} a}-e^{S_{2} b+S_{1}(a-b)}\right) \stackrel{!}{=} \frac{\left(e^{S_{2} a}-e^{S_{2} b+S_{1}(a-b)}\right) S_{1}}{e^{S_{2} b}\left(S_{1}-S_{2}\right)} .
\end{aligned}
$$

Note that according to our assumptions we have $S_{1} \neq S_{2}$. Combining those equations and rearranging terms results in

$$
0 \stackrel{!}{=} \phi\left(S_{2}-S_{1}\right)+S_{1} e^{S_{2}(a-b)}-S_{2} e^{S_{1}(a-b)} .
$$

For $h \geq 0$ define $H(h):=\phi\left(S_{2}-S_{1}\right)+S_{1} e^{-S_{2} h}-S_{2} e^{-S_{1} h}$, then we have that $H(0)=(\phi-1)\left(S_{2}-S_{1}\right)>0$, since $\phi>1, \lim _{h \rightarrow \infty} H(h)=-\infty$ and $H^{\prime}(h)<0$ for $h>0$, which means that there exists a unique $\bar{h}>0$ such that the equation is fulfilled.

Further it holds that if $1<\phi<\tilde{V}^{\prime}(0, \tilde{b})$ then $0<\bar{h}<\tilde{b}$. Namely if there would exist an $h \geq \tilde{b}>0$ such that $H(h)=0$ then we would have

$$
\phi=\frac{S_{2} e^{-S_{1} h}-S_{1} e^{-S_{2} h}}{\left(S_{2}-S_{1}\right)} \geq \frac{S_{2} e^{-S_{1} \tilde{b}}-S_{1} e^{-S_{2} \tilde{b}}}{\left(S_{2}-S_{1}\right)}=\tilde{V}^{\prime}(0, \tilde{b}),
$$

since the term on the left hand side of the inequality is strictly monotonically increasing in $h$ for all $h>0$. But this is a contradiction to the assumption for $\phi$. On top of this note that if $\phi=1$ or $\phi=\tilde{V}^{\prime}(0, \tilde{b})$ then we obtain that $\bar{h}=0$ or $\bar{h}=\tilde{b}$ respectively, which is in line with the former investigations.

Finally, it remains to prove that for this given $\bar{h}$ there exists an $a$ such that

$$
V_{u}^{\prime \prime}(a+\bar{h} ; a, a+\bar{h})=B_{1}(a, a+\bar{h}) S_{1}^{2} e^{S_{1}(a+\bar{h})}+B_{2}(a, a+\bar{h}) S_{2}^{2} e^{S_{2}(a+\bar{h})} \stackrel{!}{=} 0 .
$$

For this purpose we plug $\phi=\frac{S_{2} e^{-S_{1} \bar{h}}-S_{1} e^{-S_{2} \bar{h}}}{\left(S_{2}-S_{1}\right)}$ into this equation in order to work with the correct value for $h$. We obtain that 


$$
\left.V_{u}^{\prime \prime}(a+\bar{h} ; a, a+\bar{h})\right|_{a=0}=\frac{S_{1}^{2} e^{\bar{h} S_{1}}\left(\alpha+S_{1}\right)-S_{2}^{2} e^{\bar{h} S_{2}}\left(\alpha+S_{2}\right)}{S_{1} e^{\bar{h} S_{1}}\left(\alpha+S_{1}\right)-S_{2} e^{\bar{h} S_{2}}\left(\alpha+S_{2}\right)}<0,
$$

since $\bar{h}<\tilde{b}$. On the other hand, if we let $a$ tend to infinity we get that

$$
\lim _{a \rightarrow \infty} V_{u}^{\prime \prime}(a+\bar{h} ; a, a+\bar{h}):=C(\bar{h})>0 .
$$

This holds true since

$$
C(\bar{h})=\frac{\alpha P(\beta+\delta)+W S_{1} S_{2}^{2}-e^{\bar{h}\left(S_{1}-S_{2}\right)}\left(\alpha Q(\beta+\delta)+W S_{1}^{2} S_{2}\right)}{\alpha(\beta+\delta)\left(\frac{P}{S_{2}}-e^{\bar{h}\left(S_{1}-S_{2}\right)} \frac{Q}{S_{1}}\right)},
$$

where

$$
\begin{aligned}
P:= & S_{2}^{2}\left(\alpha+S_{2}\right)\left(\alpha \delta\left(R_{2}-S_{1}\right)\left(\alpha+R_{2}+S_{1}\right)-\beta S_{1}\left(\alpha+R_{2}\right)\left(\alpha+S_{1}\right)\right), \\
Q:= & S_{1}^{2}\left(\alpha+S_{1}\right)\left(\alpha \delta\left(R_{2}-S_{2}\right)\left(\alpha+R_{2}+S_{2}\right)-\beta S_{2}\left(\alpha+R_{2}\right)\left(\alpha+S_{2}\right)\right), \\
W:= & \beta\left(\alpha+S_{1}\right)\left(\alpha+S_{2}\right) \times \\
& \times\left(\alpha^{2}(\beta+\delta)-\alpha c R_{2}\left(\alpha+R_{2}\right)+R_{2}^{2}(\delta+\lambda)+\alpha R_{2}(\beta+\delta+\lambda)\right) .
\end{aligned}
$$

If we interpret $C$ as a function in $\bar{h}$ and evaluate it in zero, we see that $C(0)=\frac{\delta}{\beta+\delta} R_{2}>0$. Using this and the continuity of $C(\bar{h})$ we obtain that $\exists \epsilon>0$ such that $C(\epsilon)>0$. On top of this it even holds that $\frac{\partial}{\partial \bar{h}} C(\bar{h})=0$, which implies that $C(\bar{h})>0$ for all $\bar{h} \geq 0$. Note that the denominator of $C(\bar{h})$ is strictly positive.

Finally, since the limit is positive, there exists an $a^{*}$ such that $V_{u}^{\prime \prime}\left(a^{*}+\bar{h}\right)=0$, moreover note that $a^{*}>0$ since $\bar{h}<\tilde{b}$, if $\bar{h}=\tilde{b}$ then $a^{*}=0$. If there exists more than one $a^{*}$ such that this identity holds we decide to choose the smallest one, due to the shape of the function $V_{l}$. At this point, for $1<\phi<\tilde{V}^{\prime}(0, \tilde{b})$, we know there exists $a^{*}>0$ and $b^{*}:=a^{*}+\bar{h}>a^{*}$ such that the second order smooth fit conditions are fulfilled. Hence, the function

$$
V\left(x ; a^{*}, b^{*}\right)= \begin{cases}V_{l}\left(x ; a^{*}, b^{*}\right), & \text { if } \quad 0 \leq x \leq a^{*}, \\ V_{u}\left(x ; a^{*}, b^{*}\right), & \text { if } a^{*} \leq x \leq b^{*}, \\ x-b^{*}+V\left(b^{*} ; a^{*}, b^{*}\right), & \text { if } x>b^{*}\end{cases}
$$

is twice continuously differentiable.

As a next step we have to make sure that indeed our constructed function solves the HJB-equation and is concave.

First of all we obtain that for the coefficients of $V_{u}\left(x ; a^{*}, b^{*}\right)$ it holds that $B_{1}\left(a^{*}, b^{*}\right)<0$ and $B_{2}\left(a^{*}, b^{*}\right)>0$. This is valid, since if we plug the equation 


$$
B_{1}\left(a^{*}, b^{*}\right) S_{1}^{2} e^{S_{1} b^{*}}=S_{1}\left(1-B_{2}\left(a^{*}, b^{*}\right) S_{2} e^{S_{2} b^{*}}\right)
$$

into the equation $V_{u}^{\prime \prime}\left(b^{*} ; a^{*}, b^{*}\right)=0$ and rearrange some terms, we obtain that $B_{2}\left(a^{*}, b^{*}\right)>0, B_{1}\left(a^{*}, b^{*}\right)<0$ follows analogously. This directly implies that $V_{u}^{\prime \prime \prime}\left(x ; a^{*}, b^{*}\right)>0$ for all $x \geq 0$, together with $V_{u}^{\prime \prime}\left(b^{*} ; a^{*}, b^{*}\right)=0$ we get that $V_{u}^{\prime \prime}\left(x ; a^{*}, b^{*}\right)<0$ for all $x<b^{*}$, and this together with $V_{u}^{\prime}\left(b^{*} ; a^{*}, b^{*}\right)=1$ yields that $V_{u}^{\prime}\left(x ; a^{*}, b^{*}\right)>1$ for all $a^{*} \leq x<b^{*}$.

Furthermore, the first coefficient of $V_{l}\left(x ; a^{*}, b^{*}\right)$ satisfies that $A_{1}\left(a^{*}, b^{*}\right)<0$, since we can use the identity

$$
A_{2}\left(a^{*}, b^{*}\right) R_{2}^{2} e^{R_{2} a^{*}}=R_{2}\left(\phi-A_{1}\left(a^{*}, b^{*}\right) R_{1} e^{R_{1} a^{*}}-A_{3}\left(a^{*}, b^{*}\right)\right),
$$

in order to derive from the inequality $V_{l}^{\prime \prime}\left(a^{*} ; a^{*}, b^{*}\right)=V_{u}^{\prime \prime}\left(a^{*} ; a^{*}, b^{*}\right)<0$ that $A_{1}\left(a^{*}, b^{*}\right)<0$. Knowing that $A_{3}\left(a^{*}, b^{*}\right)>0$, we distinguish between the following cases, namely if $A_{2}\left(a^{*}, b^{*}\right)<0$ then $V_{l}^{\prime \prime}\left(x ; a^{*}, b^{*}\right)<0$ for all $0 \leq x \leq a^{*}$ and this property together with $V_{l}^{\prime}\left(a^{*} ; a^{*}, b^{*}\right)=\phi>1$ yields that $V_{l}^{\prime}\left(x ; a^{*}, b^{*}\right) \geq \phi>1$ for all $0 \leq x \leq a^{*}$.

If $A_{2}\left(a^{*}, b^{*}\right)>0$, then $V_{l}^{\prime \prime \prime}\left(x ; a^{*}, b^{*}\right)>0$ for all $0 \leq x \leq a^{*}$, which implies that $V_{l}^{\prime \prime}\left(x ; a^{*}, b^{*}\right)<0$ for all $0 \leq x \leq a^{*}$, since $V_{l}^{\prime \prime}\left(a^{*} ; a^{*}, b^{*}\right)=V_{u}^{\prime \prime}\left(a^{*} ; a^{*}, b^{*}\right)<0$. Now the concavity together with $V_{l}^{\prime}\left(a^{*} ; a^{*}, b^{*}\right)=\phi>1$ yields that $V_{l}^{\prime}\left(x ; a^{*}, b^{*}\right) \geq \phi>1$ for all $0 \leq x \leq a^{*}$. Additionally, we can deduce that $V\left(x ; a^{*}, b^{*}\right)>\frac{\phi c}{\delta+\lambda}>0$. This can be shown as already done in Lemma 1 , just by using the equation for $V_{l}\left(x ; a^{*}, b^{*}\right)$ in $x=0$ and exploiting that $\beta\left(V_{l}\left(a^{*} ; a^{*}, b^{*}\right)-V_{l}\left(0 ; a^{*}, b^{*}\right)-\phi a^{*}\right)>0$, due to concavity and $V_{l}^{\prime}\left(a^{*} ; a^{*}, b^{*}\right)=\phi$.

In the end, if we insert the function $V\left(x ; a^{*}, b^{*}\right)$ into the HJB-equation we obtain that for $x \in\left[0, a^{*}\right]$ the first part of the HJB-equation is zero and the second part is less than zero. For $x \in\left(a^{*}, b^{*}\right]$ the same holds true, since the supremum term in the first part is zero, because

$$
\begin{aligned}
& V\left(x+f ; a^{*}, b^{*}\right)=V\left(x ; a^{*}, b^{*}\right)+V^{\prime}\left(x ; a^{*}, b^{*}\right) f+V^{\prime \prime}\left(\theta ; a^{*}, b^{*}\right) \frac{1}{2} f^{2} \\
& \quad<V\left(x ; a^{*}, b^{*}\right)+\phi f,
\end{aligned}
$$

holds true, for a $\theta \in(x, x+f)$, provided that $f>0$, otherwise if $f=0$ the supremum part is also zero.

For $x>b^{*}$ we have to show that the second part of the HJB-equation is zero and the first part is less than zero. For that reason we consider the function

$$
\begin{aligned}
q(x):= & c V^{\prime}\left(x ; a^{*}, b^{*}\right)-(\lambda+\delta)+\lambda \int_{0}^{x} V\left(x-y ; a^{*}, b^{*}\right) \alpha e^{-\alpha y} d y \\
& +\beta \sup _{f \geq 0}\left\{V\left(x+f ; a^{*}, b^{*}\right)-V\left(x ; a^{*}, b^{*}\right)-\phi f\right\}, \quad x>b^{*} .
\end{aligned}
$$

We have to show that $q(x)<0$ for all $x>b^{*}$. We already know that $q\left(b^{*}\right)=0$ and that the supremum part in $q(x)$ is zero, since $V\left(x ; a^{*}, b^{*}\right)$ is linear for $x>b^{*}$ and $\phi>1$. Furthermore, we use the properties of the coefficients of $V_{l}\left(x ; a^{*}, b^{*}\right)$ and 
$V_{u}\left(x ; a^{*}, b^{*}\right)$. Together with the smooth fit conditions (12) and (13) we get, surprisingly nice,

$$
\begin{aligned}
& B_{1}\left(a^{*}, b^{*}\right)=\frac{S_{2} \phi e^{b^{*} S_{2}}}{S_{1} S_{2} e^{a^{*} S_{1}+b^{*} S_{2}}-S_{1}^{2} e^{a^{*} S_{2}+b^{*} S_{1}}}, \\
& B_{2}\left(a^{*}, b^{*}\right)=\frac{S_{1} \phi e^{b^{*} S_{1}}}{S_{1} S_{2} e^{a^{*} S_{2}+b^{*} S_{1}}-S_{2}^{2} e^{a^{*} S_{1}+b^{*} S_{2}}} .
\end{aligned}
$$

In addition to that, we use the identity for $\phi$ given in (15) to obtain

$$
q^{\prime}(x)=\left(e^{\left(b^{*}-x\right) \alpha}-1\right) \delta<0, \text { for all } x>b^{*} .
$$

Finally, this yields that $q(x)<0$ for $x>b^{*}$, which verifies that $V\left(x ; a^{*}, b^{*}\right)$ satisfies the first part of the HJB-equation for $x>b^{*}$. Obviously, the function $V\left(x ; a^{*}, b^{*}\right)$ satisfies $1-V^{\prime}\left(x ; a^{*}, b^{*}\right)=0$ for $x>b^{*}$ and this shows that $V\left(x ; a^{*}, b^{*}\right)$ solves the second part of the HJB-equation. Overall, this means that the function specified in (16) solves the HJB-eq. (3).

\section{Verification Theorem}

Here we state a verification theorem which fits to our constructed function $V\left(x ; a^{*}, b^{*}\right)$ in (11), (16) and Proposition 1.

Theorem 2 Let $g \in \mathcal{C}^{2}(0, \infty)$ be a positive solution to the HJB-equation

$$
\begin{aligned}
\max & \left\{c g^{\prime}(x)-(\lambda+\delta) g(x)+\lambda \int_{0}^{x} g(x-y) d F_{Y}(y)\right. \\
& \left.+\beta \sup _{f \geq 0}\{g(x+f)-g(x)-\phi f\}, 1-g^{\prime}(x)\right\}=0,
\end{aligned}
$$

where $F_{Y}(x)=\left(1-e^{-\alpha x}\right) I_{\{x \geq 0\}}$. We set $g(x)=0$, if $x<0$. Further let $g$ be concave, then

$$
g(x) \geq V(x),
$$

where

$$
V(x)=\sup _{(L, f) \in \Theta} \mathbb{E}_{x}\left[\int_{0}^{\tau^{L, f}} e^{-\delta t} d L_{t}-\phi \int_{0}^{\tau^{L, f}} e^{-\delta t} f_{t} d B_{t}\right]
$$


and $\tau^{L, f}=\inf \left\{t \geq 0 \mid X_{t}^{L, f}<0\right\}$.

Proof Let $g \in C^{2}(0, \infty)$ and $(L, f)$ be an admissible control strategy. In the following we will denote the state process $X_{t}^{L, f}$ depending on $(L, f)$ with $X_{t}$ and $\tau^{L, f}$ with $\tau$ for the sake of clarity. Because we want to make use of important theorems from stochastic calculus we have to switch to the right-continuous process, see also Shreve et al. [12, p.60-62]. We consider the process

$$
Y_{t}=e^{-\delta(t \wedge \tau)} g\left(\bar{X}_{t \wedge \tau}\right)+\int_{0}^{t \wedge \tau} e^{-\delta s} d L_{s+}-\phi \int_{0}^{t \wedge \tau} e^{-\delta s} f_{s} d B_{s},
$$

where $\bar{X}_{t}:=X_{t+}$. First of all we apply the integration by parts formula to the first part of $Y$ and Itô's formula for $\bar{X}_{s}$. We get

$$
\begin{aligned}
e^{-\delta(t \wedge \tau)} g\left(\bar{X}_{t}^{\tau}\right)=g\left(X_{0+}\right)+\int_{0+}^{t \wedge \tau} e^{-\delta s}\left[-\delta g\left(\bar{X}_{s-}\right)+c g^{\prime}\left(\bar{X}_{s-}\right)\right] d s \\
-\int_{0+}^{t \wedge \tau} e^{-\delta s} g^{\prime}\left(\bar{X}_{s-}\right) d L_{s}^{c}+\sum_{0<s \leq t \wedge \tau} e^{-\delta s} \Delta g\left(\bar{X}_{s-}\right) .
\end{aligned}
$$

Moreover, we can split up the above sum of the discontinuous parts such that we obtain

$$
\begin{aligned}
\sum_{0<s \leq t \wedge \tau} e^{-\delta s} \Delta g\left(\bar{X}_{s-}\right)= & \sum_{0<s \leq t \wedge \tau, L_{s+} \neq L_{s}} e^{-\delta s}\left[g\left(\bar{X}_{s-}-\Delta L_{s+}\right)-g\left(\bar{X}_{s-}\right)\right] \\
& +\sum_{0<s \leq t \wedge \tau, S_{s} \neq S_{s-}} e^{-\delta s}\left[g\left(\bar{X}_{s-}-Y_{N_{s}}\right)-g\left(\bar{X}_{s-}\right)\right] \\
& +\sum_{0<s \leq t \wedge \tau, B_{s} \neq B_{s-}} e^{-\delta s}\left[g\left(\bar{X}_{s-}+f_{s}\right)-g\left(\bar{X}_{s-}\right)\right] .
\end{aligned}
$$

As in [4, p. 19 - 20] with $g^{\prime} \geq 1$, we obtain for the sum belonging to the jumps of the dividend process the estimate

$$
\begin{aligned}
& \sum_{0<s \leq t \wedge \tau, L_{s+} \neq L_{s}} e^{-\delta s}\left[g\left(\bar{X}_{s-}-\Delta L_{s+}\right)-g\left(\bar{X}_{s-}\right)\right] \\
= & -\sum_{0<s \leq t \wedge \tau, L_{s+} \neq L_{s}} e^{-\delta s} \int_{0+}^{\Delta L_{s+}} g^{\prime}\left(\bar{X}_{s-}-u\right) d u \leq-\sum_{0<s \leq t \wedge \tau, L_{s+} \neq L_{s}} e^{-\delta s} \Delta L_{s+} .
\end{aligned}
$$

For the sums comprising the other jumps we take expectations and use the compensation formula to get 


$$
\begin{aligned}
& \mathbb{E}_{x}\left[\sum_{0<s \leq t \wedge \tau, S_{s} \neq S_{s-}} e^{-\delta s}\left[g\left(\bar{X}_{s-}-Y_{N_{s}}\right)-g\left(\bar{X}_{s-}\right)\right]\right] \\
& \quad=\mathbb{E}_{x}\left[\int_{0+}^{t \wedge \tau} e^{-\delta s}\left(\lambda \int_{0}^{\bar{X}_{s-}} g\left(\bar{X}_{s-}-y\right) d F_{Y}(y)-\lambda g\left(\bar{X}_{s-}\right)\right) d s\right]
\end{aligned}
$$

and

$$
\begin{aligned}
\mathbb{E}_{x}\left[\sum_{0<s \leq t \wedge \tau, B_{s} \neq B_{s-}} e^{-\delta s}\left[g\left(\bar{X}_{s-}+f_{s}\right)-g\left(\bar{X}_{s-}\right)\right]\right] \\
\quad=\mathbb{E}_{x}\left[\int_{0+}^{t \wedge \tau} e^{-\delta s} \beta\left[g\left(\bar{X}_{s-}+f_{s}\right)-g\left(\bar{X}_{s-}\right)\right] d s\right] .
\end{aligned}
$$

Now, we exploit the results from above to obtain

$$
\begin{aligned}
\mathbb{E}_{x}\left[e^{-\delta(t \wedge \tau)} g\left(\bar{X}_{t}^{\tau}\right)\right] \leq & \mathbb{E}_{x}\left[g\left(X_{0+}\right)+\int_{0+}^{t \wedge \tau} e^{-\delta s}\left[-\delta g\left(\bar{X}_{s-}\right)+c g^{\prime}\left(\bar{X}_{s-}\right)\right] d s\right. \\
& +\int_{0+}^{t \wedge \tau} e^{-\delta s}\left(\lambda \int_{0}^{\bar{X}_{s-}} g\left(\bar{X}_{s-}-y\right) d F_{Y}(y)-\lambda g\left(\bar{X}_{s-}\right)\right) d s \\
& \left.+\int_{0+}^{t \wedge \tau} e^{-\delta s} \beta\left[g\left(\bar{X}_{s-}+f_{s}\right)-g\left(\bar{X}_{s-}\right)\right] d s-\int_{0+}^{t \wedge \tau} e^{-\delta s} d L_{s+}\right] .
\end{aligned}
$$

Next we use that $g$ solves the HJB-equation

$$
\mathbb{E}_{x}\left[e^{-\delta(t \wedge \tau)} g\left(\bar{X}_{t}^{\tau}\right)\right] \leq \mathbb{E}_{x}\left[g\left(X_{0+}\right)-\int_{0+}^{t \wedge \tau} e^{-\delta s} d L_{s}+\int_{0+}^{t \wedge \tau} e^{-\delta s} \beta \phi f_{s} d s\right] .
$$

Adding on both sides $\mathbb{E}_{x}\left[\int_{0}^{t \wedge \tau} e^{-\delta s} d L_{s+}-\int_{0}^{t \wedge \tau} e^{-\delta s} \beta \phi f_{s} d s\right]$ and using the concavity of $g$ yields that

$$
\mathbb{E}_{x}\left[Y_{t}\right] \leq \mathbb{E}_{x}\left[g\left(X_{0+}\right)+\Delta L_{0+}\right] \leq g(x)
$$

The last inequality shows that the process $Y$ is a supermartingale. Now we use this property to obtain 


$$
\begin{aligned}
g(x) & =Y_{0} \geq \mathbb{E}_{x}\left(Y_{t}\right) \geq \mathbb{E}_{x}\left[\int_{0}^{t \wedge \tau} e^{-\delta s}\left(d L_{s}-\phi f_{s} d B_{s}\right)\right] \\
& \geq \mathbb{E}_{x}\left[\int_{0}^{\lfloor t\rfloor \wedge \tau} e^{-\delta s} d L_{s}\right]-\mathbb{E}_{x}\left[\int_{0}^{(\lfloor t\rfloor+1) \wedge \tau} e^{-\delta s} \phi f_{s} d B_{s}\right],
\end{aligned}
$$

where we exploited that $g \geq 0$. Considering the limit $t \rightarrow \infty$ and using monotone convergence gives

$$
g(x) \geq \mathbb{E}_{x}\left[\int_{0}^{\tau} e^{-\delta s}\left(d L_{s}-\phi f_{s} d B_{s}\right)\right] .
$$

Taking the supremum over all admissible strategies results in the desired relation:

$$
g(x) \geq V(x)
$$

Since for all parameter constellations our constructed functions are linked to an admissible strategy, are twice differentiable and concave, we have that they dominate the value function. Furthermore, using the band type strategy specified by $\left(a^{*}, b^{*}\right)$ we have that the corresponding $Y$ from (18) is a martingale. Instead of using dominated convergence in the limitation procedure, one can even use bounded convergence, since $f_{s}^{*} \leq a^{*}$ and observe that $\lim _{t \rightarrow \infty} \mathbb{E}_{x}\left[e^{-\delta(t \wedge \tau)} V\left(\bar{X}_{t}^{\tau} ; a^{*}, b^{*}\right)\right]=0$.

Corollary 1 If $(\delta+\lambda)^{2}<c \alpha \lambda$ and $\phi \geq 1$, the function $V\left(x ; a^{*}, b^{*}\right)$ is the value function and the corresponding band type strategy is optimal.

\section{Extremal case: $\beta \rightarrow \infty$}

A problem interesting in its own right arises if we let $\beta$ tend to infinity. This means that investors/injections are in principle available at every point in time. Consequently, the problem turns into a fully singular control problem with value function given by

$$
V^{\infty}(x)=\sup _{(L, F) \in \Theta^{\infty}} \mathbb{E}_{x}\left[\int_{0}^{\tau^{L, F}} e^{-\delta t} d L_{t}-\phi \int_{0}^{\tau^{L, F}} e^{-\delta t} d F_{t}\right]
$$

and

$$
X_{t}^{L, F}=X_{t}-L_{t}+F_{t},
$$

where $F_{t}$ has to be càglàd and corresponds to the total funding up to time $t$. Note that the considered objects such as $\Theta^{\infty}$ and $\tau^{L, F}$ are analogously defined as in the case 
with finite $\beta$. The literature tells us that the associated HJB-equation has the following form

$$
\max \left\{c g^{\prime}(x)-(\lambda+\delta) g(x)+\lambda \int_{0}^{x} g(x-y) d F_{Y}(y), 1-g^{\prime}(x), g^{\prime}(x)-\phi\right\}=0,
$$

see also [9], with the subtle difference that in our model the insurance company can face ruin, whereas in the reference shareholders are forced to compensate for negative surplus values. Since numerical examples using our explicit solution for large but finite values of $\beta$, indicate that the strategy stays of the same type with $a^{*}>0, b^{*}>a^{*}$, we can try to solve (19) explicitly using this type of strategy as a good guess. The optimal strategy is now to keep the process within $\left[a^{*}, b^{*}\right]$, which corresponds to a reflection at both thresholds. Notice that although investors are available at any point in time ruin can be caused by claims larger than $a^{*}$. The proposed value function has the form:

$$
V^{\infty}(x ; a, b)=\left\{\begin{array}{lll}
C^{\infty}+\phi x, & \text { if } 0 \leq x \leq a, \\
B_{1}^{\infty}(a, b) e^{S_{1} x}+B_{2}^{\infty}(a, b) e^{S_{2} x}, & \text { if } a \leq x \leq b, \\
x-b+V^{\infty}(b ; a, b), & \text { if } x>b .
\end{array}\right.
$$

Again, the coefficients $C^{\infty}, B_{1}^{\infty}(a, b)$ and $B_{2}^{\infty}(a, b)$ for given $a$ and $b$ can be calculated solving a linear system of equations. Furthermore, one can construct $a^{*}, b^{*}$ such that $\left.\frac{\partial}{\partial x} V^{\infty}\left(x ; a^{*}, b^{*}\right)\right|_{x=a^{*}}=\phi$ and $\left.\frac{\partial^{2}}{\partial x^{2}} V^{\infty}\left(x ; a^{*}, b^{*}\right)\right|_{x=b^{*}}=0$. The proof of existence goes along the lines of arguments as presented in Sect. 2. This leads to a concave solution $V^{\infty}\left(x ; a^{*}, b^{*}\right)$. Hence, similar verification arguments apply, which indeed verify that $V^{\infty}\left(x ; a^{*}, b^{*}\right)$ is optimal.

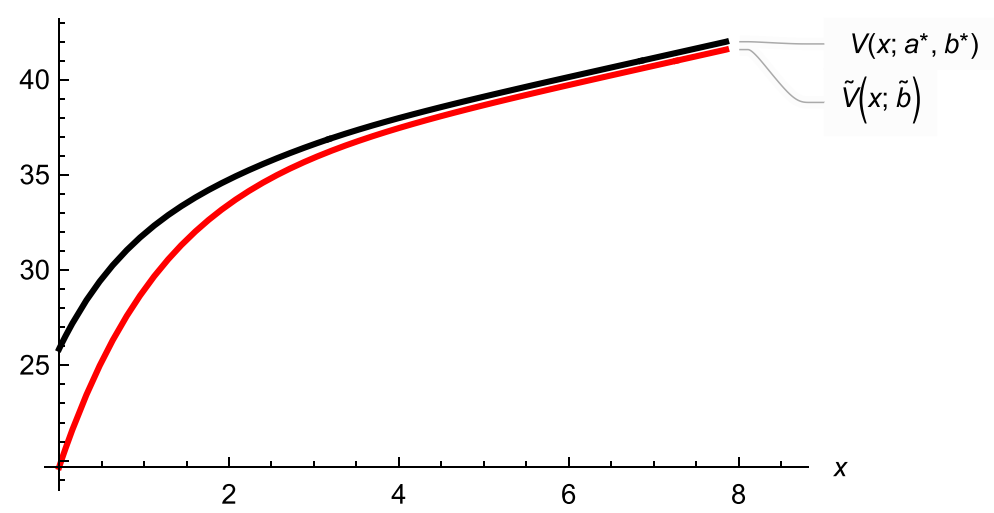

Fig. 3 Value functions 


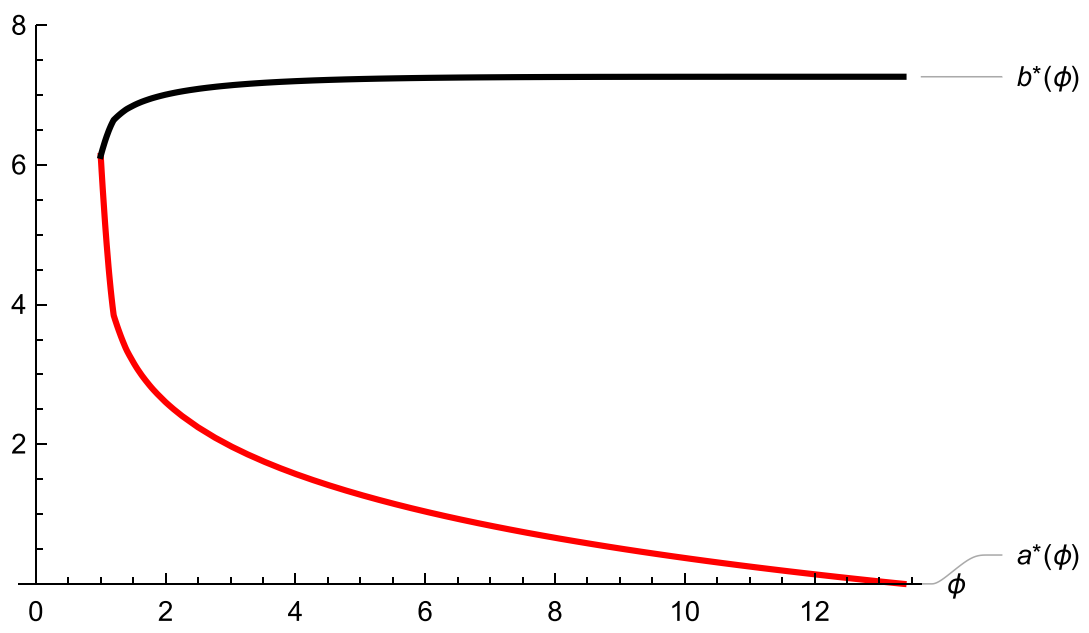

Fig. 4 Strategies as functions of $\phi$

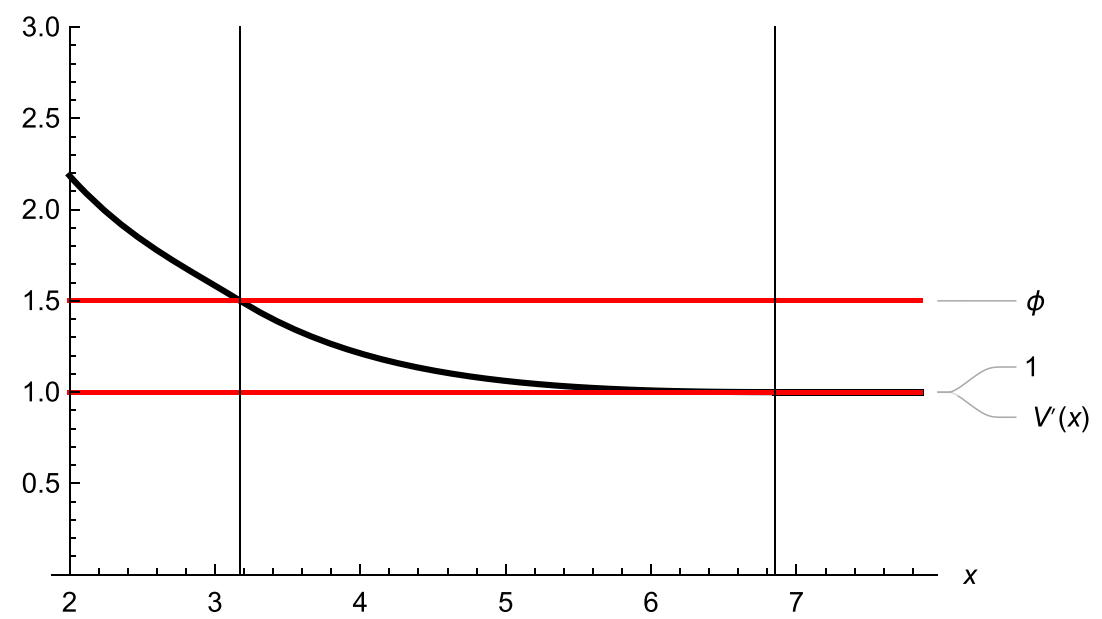

Fig. 5 1st order smooth fit

\section{Numerical illustration}

In this section we present a numerical example which nicely illustrates the dependence of the optimal strategy on the parameter $\phi \geq 1$. For this purpose we have chosen the parameters as follows. Concerning the reserve process we take $c=1.5$ for the premium rate, $\lambda=1$ for the intensity of the Poisson process associated with the claim occurrence and $\alpha=1.5$ as the parameter of the exponential distribution of the claim size. Furthermore, for the jump process $B$ we take $\beta=2$, which corresponds to the expected arrivals of investors per time unit. In terms of 


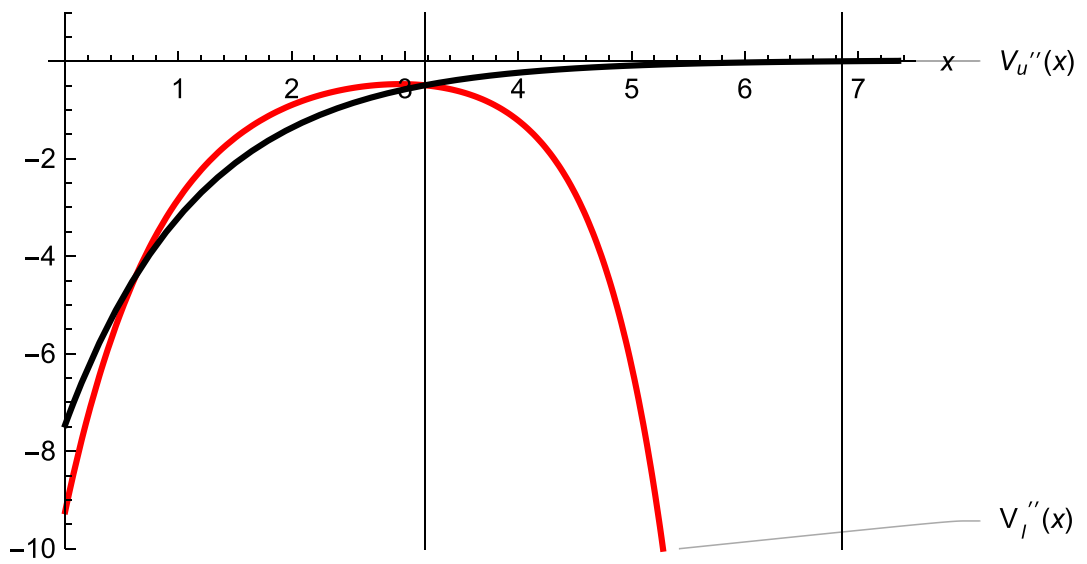

Fig. 6 2nd order smooth fit

the interest rate we choose $\delta=0.02$ and in order to illustrate the value function and the smooth fit conditions we fix $\phi=1.5$ temporarily.

In Fig. 3 we depict the difference between the value function of the usual dividend problem and the value function of the model with random capital supply with $a^{*}=3.1746, b^{*}=6.8526$. Figure 4 illustrates how the transaction cost parameter $\phi$ affects the nature of the optimal strategy in terms of $\left(a^{*}, b^{*}\right)$. As proved above, we observe that for the case $\phi=1$ the two thresholds $a^{*}$ and $b^{*}$ coincide. Further, if $\phi$ increases, the area where we search for additional funding shrinks exactly up to the certain point where it disappears. This exactly happens at $\phi=\tilde{V}^{\prime}(0 ; \tilde{b})$. Simultaneously, the dividend threshold $b^{*}$ is increasing in $\phi$ and reaches its maximum level at the point where $a^{*}$ becomes zero, namely, again if $\phi=\tilde{V}^{\prime}(0 ; \tilde{b})$. We observe that the maximum level for $b^{*}$ is the dividend barrier level $\tilde{b}$ of the usual dividend problem. On top of this we even see (and indeed proved) that for values of $\phi$ larger than $\tilde{V}^{\prime}(0 ; \tilde{b})$ the optimal strategy does not change anymore.

In the Figs. 5 and 6 we illustrate the first and second order smooth fit property. In Fig. 5 we plotted the first derivative of the value function to point out that at the lower optimal threshold $a^{*}$ we have $V^{\prime}\left(a^{*} ; a^{*}, b^{*}\right)=\phi$, which is, according to our theoretical treatment, equivalent to the second order smooth fit condition. Further, at the upper optimal threshold $b^{*}$ we have that $V^{\prime}\left(b^{*} ; a^{*}, b^{*}\right)=1$. Finally, Fig. 6 shows the second derivative of the functions $V_{l}\left(x ; a^{*}, b^{*}\right)$ and $V_{u}\left(x ; a^{*}, b^{*}\right)$ and illustrates their behaviour in the respective domain of interest.

\section{Conclusion and practical aspects}

In our contribution we introduce additional funding opportunities at random times into the classical risk model with dividends. In addition to solving the associated optimization problem, we present arguments which link the traditional injection procedure 
by initial investors to the situation of funding by external investors. Since such external funding problems are heavily discussed in corporate finance, this establishes a further link between company valuations and the optimal dividend problem in insurance. Clearly, the proposed modification leads to an increase of the value of an insurance portfolio (measured by future dividends) over the classical dividend situation. This fact is illustrated in Fig. 3. Hence, the firm value can be raised through the acceptance of capital provided by outside investors. In the case of disproportionate large transaction costs the externally appropriated funding is too costly and therefore the management will not take advantage of this possibility. Thus, the optimal strategy heavily depends on the transaction cost parameter $\phi$ as exemplified in Fig. 4.

The presence of the funding level $a^{*} \geq 0$ stands in contrast to the optimal strategy obtained by Kulenko and Schmidli [9], where the reflection needs to take place at the zero level to prevent ruin. Since in our model the insurance company is still exposed to the risk of possible ruin, the funding level $a^{*}$ serves as the lower bound of a - one may call it - stable zone $\left[a^{*}, b^{*}\right)$, where the optimal strategy tries to keep the surplus process to stay in this area. The region of surplus values larger than $b^{*}$ can be seen as comfort zone or zone of preference where the insurance company can afford to pay dividends despite the existing risk of ruin. On the contrary if the current reserve is below $a^{*}$ we are in a hazard zone and there is need for action. Hence, the management of the insurance company makes use of every possible funding in order to arrive again at the stable zone. Of course, since our reserve process is bounded from above by $b^{*}$, the ruin event is certain, due to that there exists another region, the ruin zone. In this way the obtained optimal strategy leads to a partition of the state space. In the extremal case $\beta \rightarrow \infty$ this implies that certain claims, those which do not cause ruin but take the surplus to $\left[0, a^{*}\right)$, are immediately partly refunded such that the surplus is pushed back to the stable zone. The determination of the current zone of an insurance portfolio can be a possible guidance for the evaluation of the status quo to help the actuary to stick to the principle of prudence.

Acknowledgements Open access funding provided by Graz University of Technology.

Open Access This article is distributed under the terms of the Creative Commons Attribution 4.0 International License (http://creativecommons.org/licenses/by/4.0/), which permits unrestricted use, distribution, and reproduction in any medium, provided you give appropriate credit to the original author(s) and the source, provide a link to the Creative Commons license, and indicate if changes were made.

\section{References}

1. Albrecher H, Cheung EC, Thonhauser S (2011) Randomized observation periods for the compound poisson risk model: dividends. ASTIN Bull 41(2):645-672

2. Azcue P, Muler N (2005) Optimal reinsurance and dividend distribution policies in the CramérLundberg model. Math Financ 15(2):261-308

3. Azcue P, Muler N (2014) Stochastic optimization in insurance. Springer briefs in quantitative finance. Springer, New York

4. Azcue P, Muler N (2010) Optimal investment policy and dividend payment strategy in an insurance company. Ann Appl Probab 20(4):1253-1302

5. Binmore K, Rubinstein A, Wolinsky A (1986) The Nash bargaining solution in economic modelling. RAND J Econ 17(2):176-188 
6. Gerber HU (1969) Entscheidungskriterien für den zusammengesetzten Poisson-Prozess. Schweiz Aktuarver Mitt 69(2):186-226

7. Hugonnier J, Malamud S, Morellec E (2015) Capital supply uncertainty, cash holdings, and investment. Rev Financ Stud 28(2):391-445

8. Hugonnier J, Malamud S, Morellec E (2015) Supplementary Appendix to: Capital supply uncertainty, cash holdings, and investment. https://www.epfl.ch/schools/cdm/wp-content/uploa ds/2018/08/HMM-App.pdf

9. Kulenko N, Schmidli H (2008) Optimal dividend strategies in a Cramér-Lundberg model with capital injections. Insur. Math. Econom. 43(2):270-278

10. Rolski T, Schmidli H, Schmidt V, Teugels JL (1999) Stochastic processes for insurance and finance. Wiley, New York

11. Schmidli H (2008) Stochastic control in insurance. Probability and its applications. Springer, Berlin

12. Shreve SE, Lehoczky JP, Gaver DP (1984) Optimal consumption for general diffusions with absorbing and reflecting barriers. SIAM J. Control Optim. 22(1):55-75

13. Zhang Z, Cheung EC, Yang H (2018) On the compound Poisson risk model with periodic capital injections. ASTIN Bull 48(1):435-477

Publisher's Note Springer Nature remains neutral with regard to jurisdictional claims in published maps and institutional affiliations. 